\title{
Rapid Sequence MRI Analysis of Acute Abdominal Pain
}

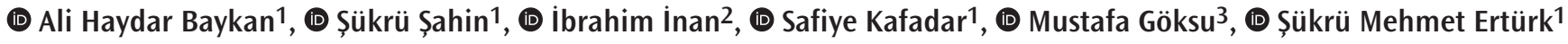 \\ 1Department of Radiology, Adıyaman University Faculty of Medicine, Adıyaman, Turkey \\ ${ }^{2}$ Clinic of Radiology, Biruni University Hospital, İstanbul, Turkey \\ ${ }^{3}$ Department of General Surgery, Adıyaman University Faculty of Medicine, Adıyaman, Turkey
}

\begin{abstract}
Aim: The aim of this study was to evaluate the diagnostic performance of non-contrast rapid sequences (RAMRI) visualization on cases who were clinically diagnosed as having acute abdominal pain (AAP).

Materials and Methods: Forty-six patients were chosen from 2850 patients who were admitted to the emergency service between January 2016 - January 2019 because of sudden onset abdominal pain and could not get a computerized tomography analysis. A 1.5 Tesla magnetic resonance (MR) device (GE Signa Hi-Speed, Milwaukee) was used for analysis. Coronal and axial T2-weighted single-shot fast spin-echo series were used as scan protocol. No intravenous, oral or rectal contrast material was used. The cases were identified as positive (+) or negative (-) by MRI. The cases who had symptoms related with AAP, were considered as positive (+), and the cases who did not have symptoms or had symptoms which were not related with AAP, were considered as negative (-).

Results: Of the patients, 26 (56.5\%), were female and 20 (43.5\%) were male. The median age was 38.65 (18-86) years. The treatment methodology was surgery for 25 cases (54\%) and conservative for 21 (46\%) cases. The operative group (surgically treated) included $24 \mathrm{MR}(+)$ and one MR (-) cases. There was a harmony between the clinic and MRI data of all members in non-surgical group. The accuracy of the study was calculated as $95.6 \%$ (44/46). The sensitivity of RAMRI was calculated as $96 \%$ for operative group and $100 \%$ for non-operative group.
\end{abstract}

Conclusion: A successful diagnostic performance was achieved by non-contrast RAMRI in cases with AAP.

Keywords: Abdomen, magnetic resonance, pain, acute, appendicitis

\section{Introduction}

Acute abdominal pain (AAP) is one of the most common reasons of emergency service applications (1). Only a few of emergency cases can be diagnosed by physical and laboratory tests (2), because the differential diagnoses of AAP are wide and the most frequently encountered reasons are acute appendicitis (AA), biliary colic, cholecystitis, diverticulitis, ileus, gastrointestinal lumen perforation, pancreatitis and renal colic (3). Thus, radiological visualization is one of the most used methods for the diagnosis of the reasons for AAP (4). According to the conformity criteria of the American College of Radiology for the cases who have fever, delocalized stomach pain and lack of surgical background, the abdomen computerized tomography (CT) is the most suitable method (4). However, this methodology is rarely preferred for children and pregnant women because of the ionized radiation and contrast material usage which has nephrotoxic effects (57). Ultrasonography (US) can be preferred because it is easily accessible, cheap and safe (5). But there is a need for an operator and the stomach-intestine gas superposition prevents achieving high quality visualization, which are the disadvantages of the method $(7,8)$. Besides, $C T$ is more sensitive than US in delocalized AAP (4).

Magnetic resonance imaging (MRI) is not frequently used for AAP. Lack of ionized radiation and high soft tissue contrast are the advantages of MRI (5). MRI methodology is limited for intense stomach pains because of long duration for analysis and possible movement artefacts. But diagnostic images can be handled for these cases by rapid sequence abdominal MRI (RAMRI) (9). The main purpose of RAMRI analysis is to keep the patient in magnetic resonance (MR) device as short as possible and handle 
the diagnostic images by preventing movement artefacts. Singleshot sequences can be used for this purpose.

Single-shot fast spin-echo (SSFSE) sequence was used in this study to evaluate the performance of non-contrast RAMRI visualization on the cases who had clinic diagnosis of AAP.

\section{Materials and Methods}

The study had a retrospective design and the local ethics committee approval was obtained (2017/6-10) and it was carried out after the approval of the study. The study was in accordance of the working principles of the Helsinki Declaration.

\section{Patients}

Forty-six patients were chosen from 2850 patients for this study who were admitted to the emergency service because of sudden onset abdominal pain and could not get a CT analysis because of pregnancy, kidney dysfunction, history of contrast material reaction or did not have enough radiological data by US. The images were saved and evaluated retrospectively from University Hospital radiology archive. Patients who had low quality images, more than 72 hours of analysis duration with RAMRI and had renal colic diagnosis were excluded from the study.

\section{MRI Protocol}

For analysis, 1.5 Tesla MR device (GE Signa Hi-Speed, Milwaukee) was used. Coronal and axial T2-weighted SSFSE series were used as scan protocol (TR/TE/NEX: 634/101/1). Thickness and gap were determined as $5 \mathrm{~mm}$ and $0.1 \mathrm{~mm}$, respectively. Additionally, axial fat-suppressed axial T2-weighted SSFSE and T2 gradient echo (GRE) were used when essential. No intravenous, oral or rectal contrast material was used. The analysis duration was shorter than 2 min. for most of the cases.

\section{Statistical Analysis}

The images were evaluated by two radiologists who had abdomen MR experience. The operation data for surgically cured patients and final clinic diagnosis for conservatively cured patients were saved. The cases were identified as positively $(+)$ and negatively (-) by MRI. The patients who had symptoms (swelling and an inflamed appendix, enlarged ovarian torsion, pericholecystic fluid and accompanier biliary gallstone, existence of free fluid, inflamed intestinal wall) related with AAP were considered as positive $(+)$; the patients who did not have symptoms or had symptoms (simple renal cortical cyst, liver hemangioma) not related with AAP were considered as negative (-).

SPSS 22.0 was used for statistical analysis. All data were managed, processed, and compiled in Microsoft Office Excel.

\section{Results}

Twenty-six (56.5\%) of the patients were female and 20 (43.5\%) of them were male. The median age was 38.65 (18-86) years. Additionally, six patients were pregnant. The treatment methodology was surgery for 25 cases (54\%) and conservative for $21(46 \%)$ patients. The operative group (surgically treated) included $24 \mathrm{MR}(+)$ and one MR (-) patients. Fifteen of these patients had $\mathrm{AA}$, three acute cholecystitis, one stomach perforation, two over torsion, 2 small bowel obstruction and one colon tumor. One of the patients who was reported as MR (-) was diagnosed as having $A A$, surgically. One of the patients who was reported as MR (+) and considered as having AA was identified as having normal appendix after surgery and pathology results. The surgical and pathologic diagnosis of other members of the operative group and MRI diagnosis of these patients were in harmony. Two of the patients were considered as having perforation, because they had periappendicular fluid and this phenomenon was proved surgically. Acute cholecystitis was evaluated in three of the patients and cholecystectomy was applied in them. Impacted cystic duct stone accompanied to one of them, cholelithiasis and choledocholithiasis accompanied to others. Perforation was considered in one of the patients because of having discontinuity at the stomach wall and free air at abdomen and this phenomenon was proved surgically (Table 1).

Three acute pancreatitis, one terminal ileitis, one colitis, one omental infarct, one pelvic inflammatory disease and one Crohn's disease were reported in the non-surgical group MR (+) which were clinically followed up and 13 cases were evaluated as non-pathologic and marked as MR (-) (Table 1). Duodenal diverticulum was diagnosed in one and gallbladder stone was detected additionally in two of the three patients who were diagnosed as having acute pancreatitis. There was a harmony between the clinic and MRI data of all members of non-surgical group. Torsion was evaluated in one of the pregnant patients because of the increase of asymmetric ovarian volume and she was detorsioned surgically. AA was diagnosed in two of the pregnant patients. No pathology was detected in the rest of the pregnant patients and the patients were discharged from hospital when the symptoms decreased. The radiological findings of some cases are shown in Figures 1 to 4.

The sensitivity of RAMRI was calculated as 96\% for operative group and 100\% for non-operative group.

\section{Discussion}

MRI is sensitive for the visualization of inflammatory changes at liver, biliary system, pancreas, urinary system, intestinal anses and pelvic organs (10). There are studies in literature which 


\begin{tabular}{|l|l|l|l|l|}
\hline \multicolumn{2}{|l|}{ Table 1. Classification of the MRI findings } \\
\hline & \multicolumn{2}{l|}{$\begin{array}{l}\text { Surgery group } \\
(\mathbf{n}=\mathbf{2 5})\end{array}$} & \multicolumn{2}{l|}{$\begin{array}{l}\text { Non-surgery } \\
\text { group }(\mathbf{n}=21)\end{array}$} \\
\hline Cases & $\begin{array}{l}\text { MR (+) } \\
(\mathbf{n}=\mathbf{2 4})\end{array}$ & $\begin{array}{l}\text { MR }(-) \\
(\mathbf{n}=\mathbf{1})\end{array}$ & $\begin{array}{l}\text { MR (+) } \\
(\mathbf{n}=\mathbf{8})\end{array}$ & $\begin{array}{l}\text { MR (-) } \\
(\mathbf{n}=13)\end{array}$ \\
\hline Appendicitis & 15 & 1 & - & - \\
\hline Cholecystitis & 3 & - & - & - \\
\hline Over torsion & 2 & - & - & - \\
\hline Intestinal obstruction & 2 & - & - & - \\
\hline Colon tumor & 1 & - & - & - \\
\hline Stomach perforation & 1 & - & - & - \\
\hline Pancreatitis & - & - & 3 & - \\
\hline Terminal ileitis & - & - & 1 & - \\
\hline Colitis & - & - & 1 & - \\
\hline Omental infarct & - & - & 1 & - \\
\hline PID & - & - & 1 & - \\
\hline Crohn & - & - & 1 & - \\
\hline $\begin{array}{l}\text { MR: Magnetic resonance, MRI: } \\
\text { inflammatory disease }\end{array}$ & Magnetic resonance imaging, PID: Pelvic \\
\hline
\end{tabular}

emphasize the activity of abdominal MRI on AA $(11,12)$, acute diverticulitis (13), acute pancreatitis (14) and acute cholecystitis (15). Nevertheless, there is a lack of literature about the MRI application in AAP. Besides, there is no study on the cost analysis (2). MRI is generally applied to the pregnant patients in whom the US is not an option. However, CT is the first option for AAP and MRI is generally considered as an alternative. It has been considered as a good alternative, especially for the pregnants and children, to prevent patients from ionized radiation (16).

$\mathrm{AA}$ is still the most common reason for AAP and the ratio of $\mathrm{AA}$ in patients with AAP is between $11-23 \%$ (17). The ratio of AA in this study was $32 \%$ (15 of 45 patients) which was higher than the literature. CT analysis was not applied to the patients and this might be a reason for the high ratio. In one case, reactive fluid increase and mild enlargement were detected by RAMRI at appendix lumen radius and periappendicular area, respectively and reported as AA. But after the surgery it was concluded that there was no AA and the appendix symptoms improved. One of the other patients was reported as MR (-) by RAMRI but surgical evidences showed that the diagnosis was AA. In both patients, the duration between the RAMRI analysis and surgery was more than 24 hours. There are still blur parts in visualization data for AA which is an inflammatory disease. New studies should be organized with higher number of patients.

The images should be handled quickly, and they should be diagnostic in patients with AAP. The sequences like SSFSE, which have short analysis duration, can be used for this purpose. The analysis duration is shorter than $2 \mathrm{~min}$. in most of the cases.

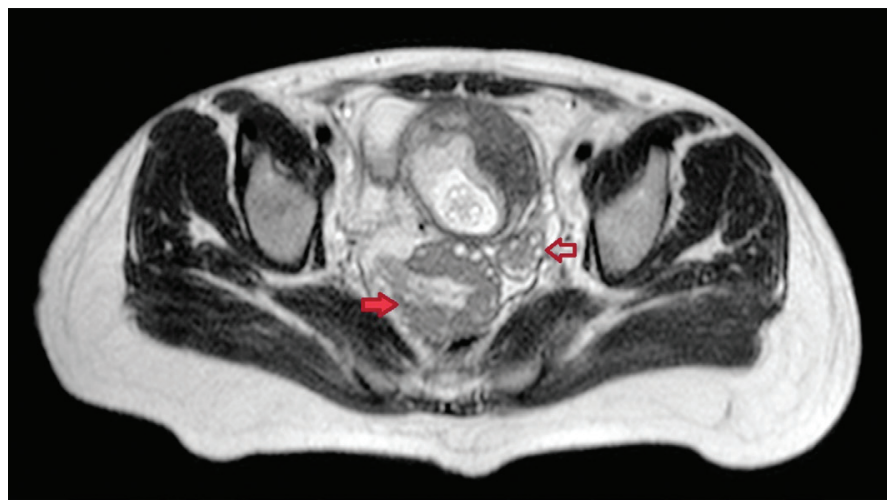

Figure 1. Increased right ovary dimensions (filled red arrow), peripherally displaced follicles and normal left ovary dimensions (empty red arrow). Right ovarian torsion

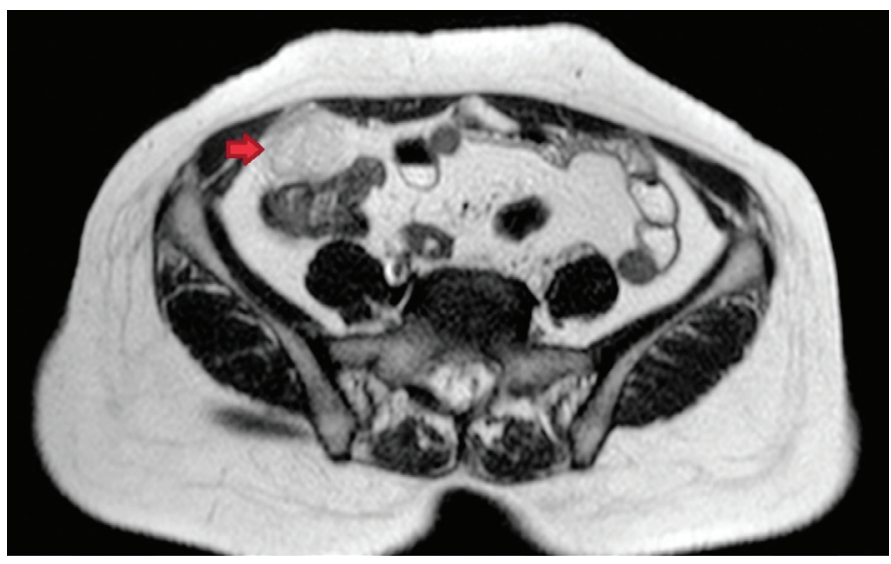

Figure 2. Inflammation at mass like fatty tissue which is at the neighborhood of anterior cecum in a patient with omental infarct

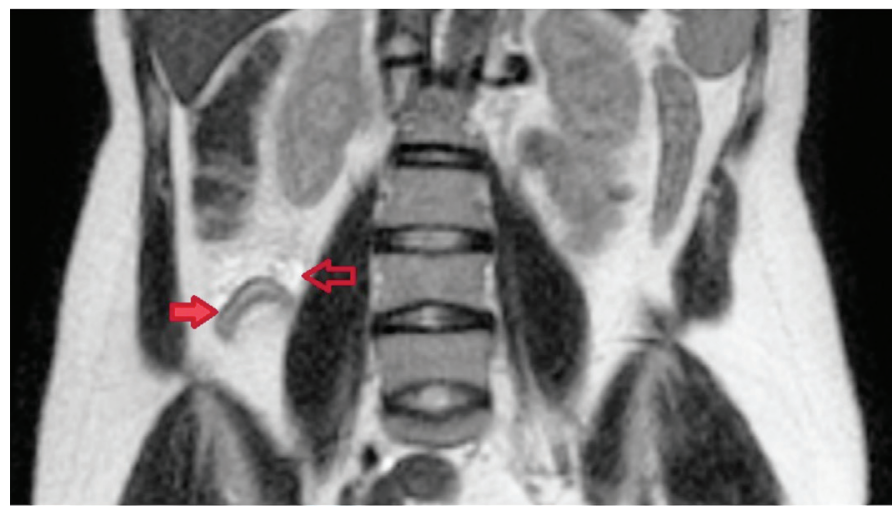

Figure 3. Dilated appendix (filled arrow) and periappendicular fluid (empty arrow) in a patient with acute appendicitis

Soft tissue resolution is considerably high and the inflammatory changes, like edema and fluid, can be diagnosed easily. It was reported that the detection sensitivity of air at GRE and inflammatory changes at T2A sequences were increased (10). These two sequences were applied in the current study when they were necessary. It was concluded that the RAMRI was significant and hopeful because the radiological analysis duration was shorter and it provided enough information. 

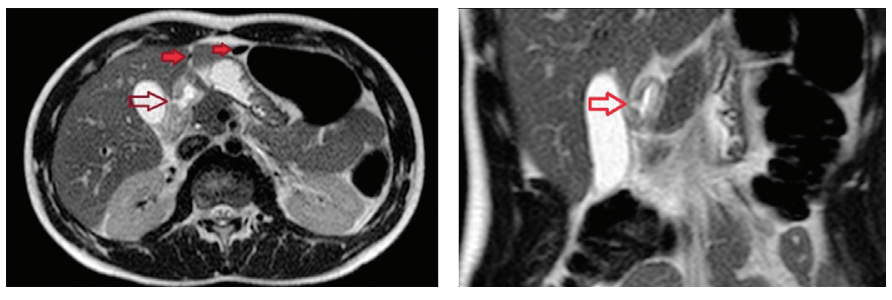

Figure 4. Free air (filled arrow) and perforation area (empty arrow) in a patient with stomach perforation

There are some disadvantages of the method; the patients who have claustrophobia and hearth battery are not allowed for MRI (17). Despite short analysis duration, diagnostic images may not be handled because of pain. The radiologist may not be familiar to the images of acute abdomen. This disadvantage can be eliminated by reputation of MRI on AA.

It was proved that rapid sequence MR was highly sensitive for the diagnosis of the AA pathology. Retrospective design of study, low patient number, absence of patients under 18 years and short follow up duration for the conservatively watched patients were the limitations of the study.

\section{Conclusion}

Rapid sequence MR is an important diagnostic tool for patients with AA when US can not provide enough data and CT is not possible. has important properties; it has high resolution for soft tissue, does not include radiation and does not need contrast material. Radiologists may not be familiar to AAP pathologies in RAMRI, because it is not a routine methodology. Frequently application of rapid sequence MRI in patients with AAP may improve the experience of radiologists.

\section{Ethics}

Ethics Committee Approval: The local ethics committee approval was obtained (2017/6-10) and it was carried out after the approval of the study. The study was in accordance of the working principles of the Helsinki Declaration.

Informed Consent: Retrospective study.

Peer-review: Externally peer-reviewed.

\section{Author Contributions}

Surgical and Medical Practices: A.H.B., Ş.Ş., I.I., S.K., M.G., Ş.M.E., Concept: A.H.B., I.I.., Design: A.H.B., Ș.Ș., S.K., Data Collection or Processing: A.H.B., S.S., M.G., S..M.E., Analysis or Interpretation: A.H.B., S.S., M.G., Literature Search: A.H.B., Ș.S., S.K., Writing: A.H.B., Ş.Ş.
Conflict of Interest: No conflict of interest was declared by the authors.

Financial Disclosure: The authors declared that this study received no financial support.

\section{References}

1. Kilicaslan I, Bozan H, Oktay C, Göksu E. Demographic properties of patients presenting to the emergency department in Turkey. Türkiye Acil Tıp Dergisi. 2005;5:5-13.

2. Stoker J, van Randen A, Laméris W, Boermeester MA. Imaging patients with acute abdominal pain. Radiology. 2009;253:31-46.

3. Abdullah M., Firmansyah MA. Diagnostic approach and management of acute abdominal pain. Acta Med Indones. 2012;44:344-50.

4. Scheirey CD, Fowler KJ, Therrien JA, Kim DH, Al-Refaie WB, Camacho MA et al ACR Appropriateness Criteria ${ }^{\circledR}$ Acute Nonlocalized Abdominal Pain. J Am Coll Radiol. 2018;15:S217-31

5. Ditkofsky NG, Singh A, Avery L, Novelline RA. The role of emergency MRI in the setting of acute abdominal pain. Emerg Radiol. 2014;21:615-24.

6. Tkacz JN, Anderson SA, Soto J. MR imaging in gastrointestinal emergencies. Radiographics. 2009;29:1767-80.

7. LeBedis CA, Penn DR, Gupta A, Tkacz JN, Jorge A et al. Current applications of MRI in emergent gastrointestinal diseases. Applied Radiology. 2012;41:9.

8. Corson-Knowles D, Russell FM. Clinical Ultrasound Is Safe and Highly Specific for Acute Appendicitis in Moderate to High Pre-test Probability Patients. West J Emerg Med. 2018;19:460-4.

9. Byott S, Harris I. Rapid acquisition axial and coronal T2 HASTE MR in the evaluation of acute abdominal pain. Eur J Radiol. 2016;85:286-90.

10. Lubarsky M, Kalb B, Sharma P, Keim SM, Martin DR. MR imaging for acute nontraumatic abdominopelvic pain: rationale and practical considerations. Radiographics. 2013;33:313-37.

11. Spalluto LB, Woodfield CA, DeBenedectis CM, Lazarus E. MR imaging evaluation of abdominal pain during pregnancy: appendicitis and other nonobstetric causes. Radiographics. 2012;32:317-34.

12. Cobben L, Groot I, Kingma L, Coerkamp E, Puylaert J, Blickman J. A simple MRI protocol in patients with clinically suspected appendicitis: results in 138 patients and effect on outcome of appendectomy. Eur Radiol. 2009;19:117583.

13. Heverhagen JT, Sitter H, Zielke A, Klose KJ. Prospective evaluation of the value of magnetic resonance imaging in suspected acute sigmoid diverticulitis. Dis Colon Rectum. 2008;51:1810-5.

14. Viremouneix L, Monneuse O, Gautier G, Gruner L, Giorgi R, Allaouchiche B et al. Prospective evaluation of nonenhanced MR imaging in acute pancreatitis. J Magn Reson Imaging. 2007;26:331-8.

15. Tonolini M, Ravelli A, Villa C, Bianco R. Urgent MRI with MR cholangiopancreatography (MRCP) of acute cholecystitis and related complications: diagnostic role and spectrum of imaging findings. Emerg Radiol. 2012;19:341-8.

16. Oto A1, Ernst RD, Shah R, Koroglu M, Chaljub G, Gei AF et al. Right-lowerquadrant pain and suspected appendicitis in pregnant women: evaluation with MR imaging--initial experience. Radiology. 2005;234:445-51.

17. Fagerström A, Paajanen P, Saarelainen $H$, Ahonen-Siirtola $M$, Ukkonen $M$, Miettinen $P$ et al. Non-specific abdominal pain remains as the most common reason for acute abdomen: 26-year retrospective audit in one emergency unit. Scand J Gastroenterol. 2017;52:1072-7. 